\title{
Efecto antifúngico de quitosán de alto peso molecular en cepas de Candida sp aisladas de muestras clínicas
}

\author{
Cecilia Tapia P., Diego Soto M., Leonardo Vergara G., Claudio Alburquerque O., \\ Andrea Maccioni R., Ana M. Matamala C., Germán Hermosilla D. y Sergio Bucarey V.
}

\section{Antifungal effect of high molecular weight chitosan on Candida spp isolated from clinical samples}

Chitosan is a D-glucosamine polysaccharide derived from chitin that displays an antimicrobial activity against bacteria and fungi. Objective: to evaluate the antifungal effect of high molecular weight chitosan (HMWC) in clinical strains of Candida spp. Methodology: the susceptibility of forty strains of Candida spp. to HMWC was studied (16 C. albicans, 11 C. glabrata, 5 C. tropicalis, 5 C. krusei, 2 C. parapsilosis and 2 C. famata) by broth microdilution at $\mathrm{pH} 7.0$ and $\mathrm{pH} 4.0$. Results: of 40 strains, only 2 were inhibited at $\mathrm{pH} 7.0$ and corresponded to ATCC control strains (C. krusei 6258 and C. parapsilosis 22019). On the other hand, 37/40 strains (92.5\%) were inhibited by concentrations lower than $1.25 \mathrm{mg} / \mathrm{mL}$ of HMWC at $\mathrm{pH} 4.0$. Conclusion: these results show that HMWC, presents activity against clinical Candida spp. strains, including C. glabrata, and that this activity is present at acid $\mathrm{pH}$ (4.0). This compound could potentially be used in vulvovaginal candidiasis since it occurs at pH 4.0-4.5.

Key words: Chitosan, antifungal effect, vulvovaginal candidiasis.

Palabras clave: Quitosán, quitosano, efecto antifúngico, candidiasis vulvovaginal.

\section{Introducción}

$\mathrm{E}$ 1 quitosán es un polímero lineal de $\beta$-1,4-Dglucosamina, derivado de la des-acetilación de la quitina (Figura 1), la cual se encuentra ampliamente distribuida en la naturaleza y forma parte estructural de insectos, crustáceos e incluso hongos ${ }^{1,2}$.

De acuerdo al grado de polimerización, el quitosán se clasifica en de bajo peso molecular (QBPM) y de alto peso molecular (QAPM).

Se le han asociado diversas propiedades terapéuticas, fundamentalmente como hipoglicemiante ${ }^{3}$, hipolipemian$\mathrm{te}^{3,4}$, para reducir sobrepeso y obesidad ${ }^{5}$ o como biopiel en pacientes con quemaduras ${ }^{6}$. Es un compuesto seguro, biocompatible y biodegradable ${ }^{7,8}$. De esta manera, es un compuesto que se ha utilizado tanto por vía oral, como por vía tópica.

Recientemente se ha descrito una actividad antimicrobiana de quitosán frente a bacterias y hongos ${ }^{1,9,10}$.

Quitosán es un compuesto insoluble en agua, pero soluble en ácidos orgánicos diluidos, como ácido acético, ácido succínico y ácido fórmico, siendo su pKa de 6,3 $3^{1}$. Estudios previos en nuestro laboratorio indican que concentraciones inferiores o iguales al 0,1\% de ácido acético, permiten diluir el quitosán sin afectar el crecimiento de levaduras (datos no mostrados).

Se ha descrito que QBPM es más activo frente a hongos ${ }^{9}$; sin embargo, un estudio reciente demostró que el QAPM tendría mayor actividad anti-fúngica debido probablemente al mayor grado de protonación de la molécula ${ }^{11}$. Ensayos preliminares en nuestro laboratorio habían demostrado actividad anti-fúngica de QBPM sobre cepas de Candida sp (datos no mostrados), por lo que se decidió evaluar la actividad anti-fúngica de QAPM en cepas clínicas de Candida sp mediante los métodos de recuento de colonias viables en placa post exposición a QAPM y microdilución en caldo.

\section{Materiales y Métodos}

Cepas: Se estudiaron 38 cepas de Candida sp provenientes de un cepario del Laboratorio de Clínica Dávila previamente identificadas a nivel de especie, mediante la metodología convencional (tubo germinativo, microcultivo) y el sistema de asimilación de azúcares API 32C AUX $^{\mathrm{TM}}$ (Biomerieux). Las cepas correspondieron a 16 C. albicans, 11 C. glabrata, 5 C. tropicalis, 5 C. krusei,
Universidad de Chile, Santiago

Facultad de Medicina Instituto de Ciencias Biomédicas Programa de Microbiología y Micología (CTP, DSM, LVG, CAO, AMR, AMMC, GHD)

Facultad de Ciencias Veterinarias y Pecuarias, Departamento de Ciencias Biológicas Animales, (SBV) Clínica Dávila, Santiago, Chile Laboratorio de Microbiología (CTP, CAO)

No existen conflictos de interés relacionados a este trabajo

Fuente de financiamiento: Proyecto BicentenarioConicyt-Banco Mundial Cod. PSD23

Recibido: 21 de enero de 2009 Aceptado: 22 de septiembre de 2009

Correspondencia a: Cecilia Tapia P. cetapia@med.uchile.cl 


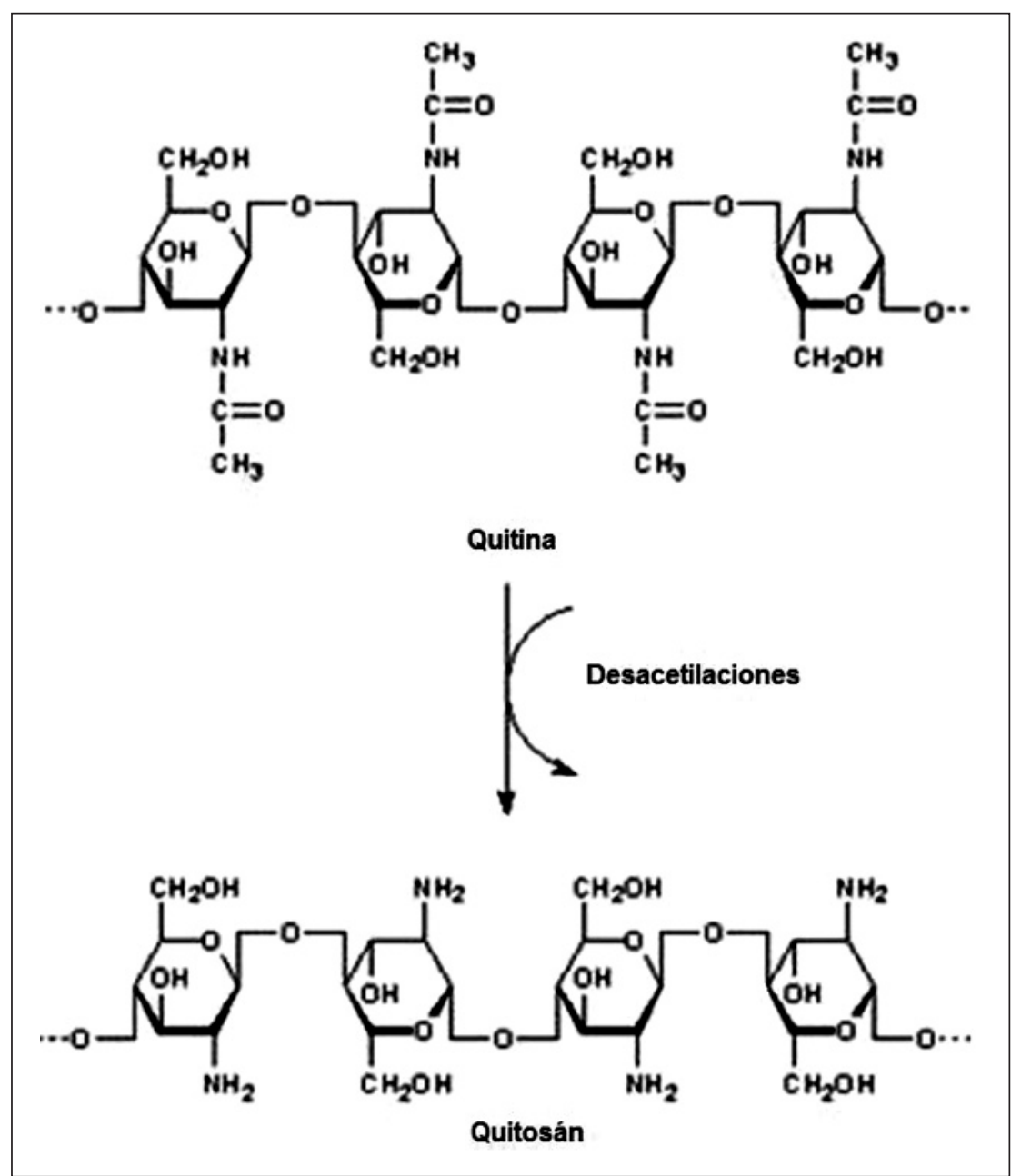

Figura 1. Desacetilación de la quitina y su producto quitosán.
2 C. parapsilosis y 2 C. famata y 3 cepas ATCC, $C$. albicans 90029, C. krusei 6258 y C. parapsilosis 22019. Se incluyeron en el estudio cepas susceptibles, susceptibles dosis dependiente (SDD) y resistentes a fluconazol. Para estudiar la susceptibilidad a QAPM se utilizaron dos métodos: recuento de colonias viables en placas post exposición a QAPM. y microdilución en caldo a $\mathrm{pH}$ 7,0 y a $\mathrm{pH} 4,0$.

Recuento de colonias viables en placas. Este método, diseñado por los autores, se realizó para evaluar preliminarmente el efecto anti-fúngico de quitosán de alto peso molecular (QAPM), Sigma-Aldrich ${ }^{\mathrm{TM}}$. El compuesto no difunde en agar, por lo que no pudo utilizarse un método de difusión en disco. Se sub-cultivaron las cepas a 37 ${ }^{\circ} \mathrm{C}$ por $24 \mathrm{hrs}$ en agar Sabouraud. Luego, se realizó una suspensión de células a una escala de Mc Farland de 0,5. Se tomaron $10 \mu \mathrm{L}$ y se agregaron a $990 \mu \mathrm{L}$ (dilución 1:100) de dos soluciones de QAPM (2 y $5 \mathrm{mg} / \mathrm{mL}$ ) diluido en ácido acético a $0,1 \%$ (mínima concentración para no inhibir el crecimiento de las levaduras).

Posteriormente se hizo una siembra en rastrillo de 100 $\mathrm{uL}$ de las soluciones de trabajo en agar Sabouraud y a las 24 hrs de incubación a $37{ }^{\circ} \mathrm{C}$ se realizó un recuento de colonias viables (Figura 2).

Adicionalmente, las cepas fueron suspendidas en otras dos soluciones, una de ácido acético solo (AA) al 0,1\% y otra de agua destilada, como controles.

Los ensayos se realizaron por duplicado.

Con el fin de evaluar el efecto temporal de QAPM, se ejecutó el mismo procedimiento anteriormente descrito, pero esta vez efectuando la siembra de tres cepas ATCC

Figura 2. Placa sembrada con cepa de Candida albicans; A) en solución de ácido acético (AA) al 0,1\%; B) en quitosán de alto peso molecular (QAPM) más AA al $0,1 \%$ post incubación a $37^{\circ} \mathrm{C}$ durante 24 hrs.

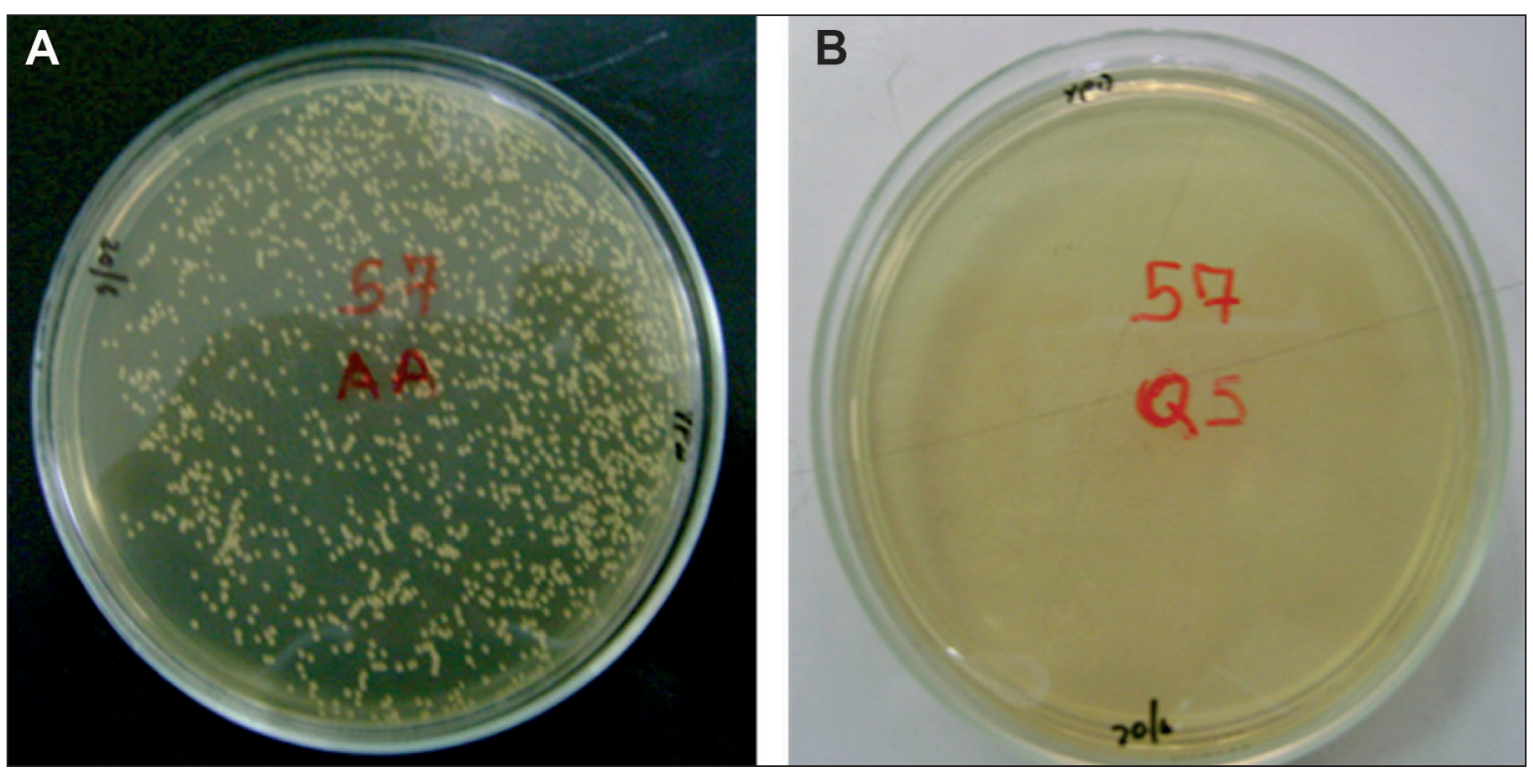


de Candida (C. albicans 90029, C. krusei 6258 y C. parapsilosis 22019) a distintos tiempos de exposición a QAPM: $1 \mathrm{~min}, 5 \mathrm{~min}, 30 \mathrm{~min}, 1 \mathrm{hr}, 2 \mathrm{hrs}$ (Tabla 2).

Microdilución en caldo: Este estudio se basó en el estándar europeo EUCAST-AFST ${ }^{12}$, con modificaciones, adaptando la técnica para medir la concentración inhibitoria mínima (CIM) a QAPM. Las modificaciones incluyen: el uso de ácido acético al 0,1\% para disolver QAPM, y la utilización de RPMI 1640 a pH 4,0, además de RPMI $1640 \mathrm{pH}$ 7,0, como medio de cultivo. El resto de las actividades se realizaron de acuerdo al estándar.

Se prepararon placas de RPMI 1640 a pH 7,0 y 4,0, con concentraciones máximas de QAPM de $1,25 \mathrm{mg} /$ $\mathrm{mL}$ (máxima concentración alcanzada que permitió la realización del ensayo).

Las cepas fueron sub-cultivadas a $37^{\circ} \mathrm{C}$ durante $24 \mathrm{hrs}$ en agar Sabouraud. Se realizó un inóculo de cada cepa alcanzando una concentración Mc Farland de 0,5. Se realizó una dilución 1:10 y se sembraron $100 \mu \mathrm{L}$ de esta dilución en las placas de microdilución. Las placas se incubaron a $35^{\circ} \mathrm{C}$ por $24 \mathrm{hrs}$, en ausencia de luz. Finalmente, se realizó una lectura espectrofotométrica de las CIMs.

\section{Resultados}

Se analizaron 21 cepas por el método de recuento de colonias viables en placa post exposición a QAPM y 40 por el de microdilución en caldo modificado.

De las cepas analizadas con el primer método, en 20/21 cepas $(95,2 \%)$ se observó una disminución del número de unidades formadoras de colonia post exposición a 2 y $5 \mathrm{mg} / \mathrm{mL}$ de QAPM (7 C. albicans, 8 C. glabrata, 3 C. parapsilosis, 1 C. tropicalis 1 C. krusei). La cepa C161 no mostró inhibición y correspondió a una $C$. glabrata (Tabla 1).

Todas las cepas presentaron inhibición al minuto de exposición a QAPM, siendo las cepas ATCC estudiadas completamente inhibidas a este tiempo (Tabla 2).

De un total de 40 cepas analizadas con el método de microdilución en caldo modificado, la mayoría de las cepas no fue inhibida por $1,25 \mathrm{mg} / \mathrm{mL}$ de QAPM a pH 7.0 (Figura 3 A). Sólo dos cepas fueron inhibidas y correspondieron a las cepas control ATCC (C. krusei y C. parapsilosis).

Por otra parte, $37 / 40(92,5 \%)$ cepas fueron inhibidas a concentraciones inferiores a $1,25 \mathrm{mg} / \mathrm{mL}$ de QAPM a $\mathrm{pH} 4.0$, incluidas las dos cepas ATCC (C. krusei y $C$. parapsilosis) (Tabla 3, Figura 3 B). Por otra parte, la mayoría de las cepas sensibles dosis dependiente (SDD) y resistentes a fluconazol, fueron inhibidas por QAPM a la concentración máxima señalada (18/21), salvo las cepas C19 (C. albicans), C144 (C. glabrata) y C161 (C.
Tabla 1. Porcentaje de inhibición de cepas de Candida sp post exposición a quitosán de alto peso molecular

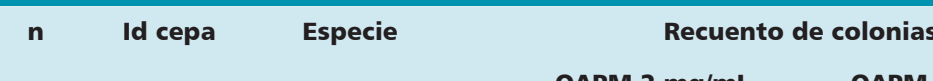

QAPM $2 \mathrm{mg} / \mathrm{mL} \quad$ QAPM $5 \mathrm{mg} / \mathrm{mL}$

(\% de inhibición) (\% de inhibición)

\begin{tabular}{|c|c|c|c|c|}
\hline 1 & $\mathrm{C} 12$ & C. albicans & 100 & 100 \\
\hline 2 & C15 & C. albicans & 100 & 100 \\
\hline 3 & C16 & C. albicans & 100 & 100 \\
\hline 4 & C26 & C. albicans & 73 & 97 \\
\hline 5 & C54 & C. albicans & 100 & 100 \\
\hline 6 & C90 & C. glabrata & 98 & 100 \\
\hline 7 & C91 & C. glabrata & 32 & 94 \\
\hline 8 & C92 & C. glabrata & 76 & 91 \\
\hline 9 & C103 & C. glabrata & 100 & 100 \\
\hline 10 & C151 & C. glabrata & 99 & 100 \\
\hline 11 & C161 & C. glabrata & 0 & 0 \\
\hline 12 & C144 & C. glabrata & 40 & 100 \\
\hline 13 & C59 & C. glabrata & 92 & 99 \\
\hline 14 & C126 & C. glabrata & 97 & 100 \\
\hline 15 & ATCC 6258 & C. krusei & 26 & 15 \\
\hline 16 & $\mathrm{C} 2$ & C. parapsilosis & 100 & 100 \\
\hline 17 & C55 & C. parapsilosis & 85 & 100 \\
\hline 18 & ATCC 22019 & C. parapsilosis & 69 & 91 \\
\hline 19 & C30 & C. tropicalis & 73 & 68 \\
\hline 20 & C57 & C. albicans & 100 & 100 \\
\hline 21 & C58 & C. albicans & 100 & 100 \\
\hline
\end{tabular}

QAPM = quitosán de alto peso molecular

Tabla 2. Perfil temporal del efecto anti-fúngico de quitosán de alto peso molecular en tres cepas ATCC de Candida sp
Cepa
No expuesta a QAPM
(ufc/100 $\mu \mathrm{L}$ )
Expuesta a QAPM
(ufc/100 $\mu \mathrm{L}$ )

\begin{tabular}{|lrrrrrr|} 
& & \multicolumn{7}{c}{ Tiempo de exposición (minutos) } \\
& & $\mathbf{1}$ & $\mathbf{5}$ & $\mathbf{3 0}$ & $\mathbf{6 0}$ & $\mathbf{1 2 0}$ \\
\hline C. albicans ATCC 90029 & 1.207 & 302 & 300 & 266 & 286 & 304 \\
C. krusei ATCC 6258 & 1.658 & 0 & 0 & 0 & 0 & 0 \\
C. parasilopsis ATCC 22019 & 1.834 & 0 & 0 & 0 & 0 & 0 \\
\hline
\end{tabular}

$\mathrm{ufc}=$ unidades formadoras de colonia. QAPM: quitosán alto peso molecular 
Tabla 3. Susceptibilidad de cepas clínicas de Candida sp a fluconazol (FCZ) y a quitosán de alto peso molecular a pH 4,0

\begin{tabular}{|c|c|c|c|c|c|}
\hline $\mathbf{n}$ & Id cepa & Especie & $\begin{array}{l}\text { CIM FCZ } \\
\mu \mathrm{g} / \mathrm{mL}\end{array}$ & $\begin{array}{c}\text { Categoría de } \\
\text { susceptibilidad a FCZ }\end{array}$ & $\begin{array}{l}\text { CIM QAPM } \\
\mathbf{m g} / \mathbf{m L}\end{array}$ \\
\hline 1 & C54 & C. albicans & 0,125 & S & 0,0025 \\
\hline 2 & $\mathrm{C} 12$ & C. albicans & 0,125 & $\mathrm{~s}$ & 0,0025 \\
\hline 3 & C16 & C. albicans & 0,125 & $\mathrm{~s}$ & 0,0025 \\
\hline 4 & C167 & C. albicans & 0,125 & $s$ & 0,005 \\
\hline 5 & C15 & C. albicans & 0,125 & $S$ & 0,625 \\
\hline 6 & C57 & C. albicans & 0,25 & $S$ & 0,0025 \\
\hline 7 & C166 & C. albicans & 0,25 & $\mathrm{~s}$ & 0,0025 \\
\hline 8 & C168 & C. albicans & 0,25 & $S$ & 0,0025 \\
\hline 1 & $\mathrm{C} 26$ & C. albicans & 0,25 & $\mathrm{~s}$ & 0,005 \\
\hline 2 & C58 & C. albicans & 0,5 & $S$ & 0,0025 \\
\hline 3 & C130 & C. albicans & 0,5 & $\mathrm{~s}$ & 0,0025 \\
\hline 4 & C129 & C. albicans & 16 & SDD & 0,0025 \\
\hline 5 & C41 & C. albicans & 64 & $\mathrm{R}$ & 0,0025 \\
\hline 6 & C62 & C. albicans & 64 & $\mathrm{R}$ & 0,0025 \\
\hline 7 & C75 & C. albicans & 64 & $\mathrm{R}$ & 0,0025 \\
\hline 8 & C43 & C. albicans & 64 & $\mathrm{R}$ & 0,005 \\
\hline 9 & $\mathrm{C} 93$ & C. albicans & 64 & $\mathrm{R}$ & 0,005 \\
\hline 10 & C94 & C. albicans & 64 & $\mathrm{R}$ & 0,005 \\
\hline 11 & C19 & C. albicans & 64 & $\mathrm{R}$ & $>1,25$ \\
\hline 12 & C113 & C. famata & 32 & SDD & 0,0025 \\
\hline 13 & C32 & C. famata & 32 & SDD & 0,005 \\
\hline 14 & C126 & C. glabrata & 16 & SDD & 0,625 \\
\hline 15 & C151 & C. glabrata & 16 & SDD & 1,25 \\
\hline 16 & C144 & C. glabrata & 16 & SDD & $>1,25$ \\
\hline 17 & C59 & C. glabrata & 32 & SDD & 0,0025 \\
\hline 18 & C92 & C. glabrata & 32 & SDD & 0,0025 \\
\hline 19 & C152 & C. glabrata & 32 & SDD & 0,0025 \\
\hline 20 & C103 & C. glabrata & 32 & SDD & 0,0025 \\
\hline 21 & C91 & C. glabrata & 32 & SDD & 0,005 \\
\hline 22 & $\mathrm{C} 44$ & C. glabrata & 32 & SDD & 0,01 \\
\hline 23 & C90 & C. glabrata & 32 & SDD & 0,01 \\
\hline 24 & C161 & C. glabrata & 64 & $\mathrm{R}$ & $>1,25$ \\
\hline 25 & $\mathrm{C} 128$ & C. krusei & 4 & S & 0,0025 \\
\hline 26 & C55 & C. parapsilosis & 0,125 & s & 0,0025 \\
\hline 26 & $\mathrm{C} 2$ & C. parapsilosis & 1 & S & 0,0025 \\
\hline 27 & C30 & C. tropicales & 0,5 & S & 0,625 \\
\hline 28 & C35 & C. tropicales & 1 & $\mathrm{~s}$ & 0,0025 \\
\hline 29 & $\mathrm{C} 14$ & C. tropicales & 4 & $S$ & 0,0025 \\
\hline 30 & ATCC 6258 & C. krusei & 8 & $S$ & 0,0025 \\
\hline 31 & ATCC 22019 & C. parapsilosis & 1 & $\mathrm{~s}$ & 0,0025 \\
\hline
\end{tabular}

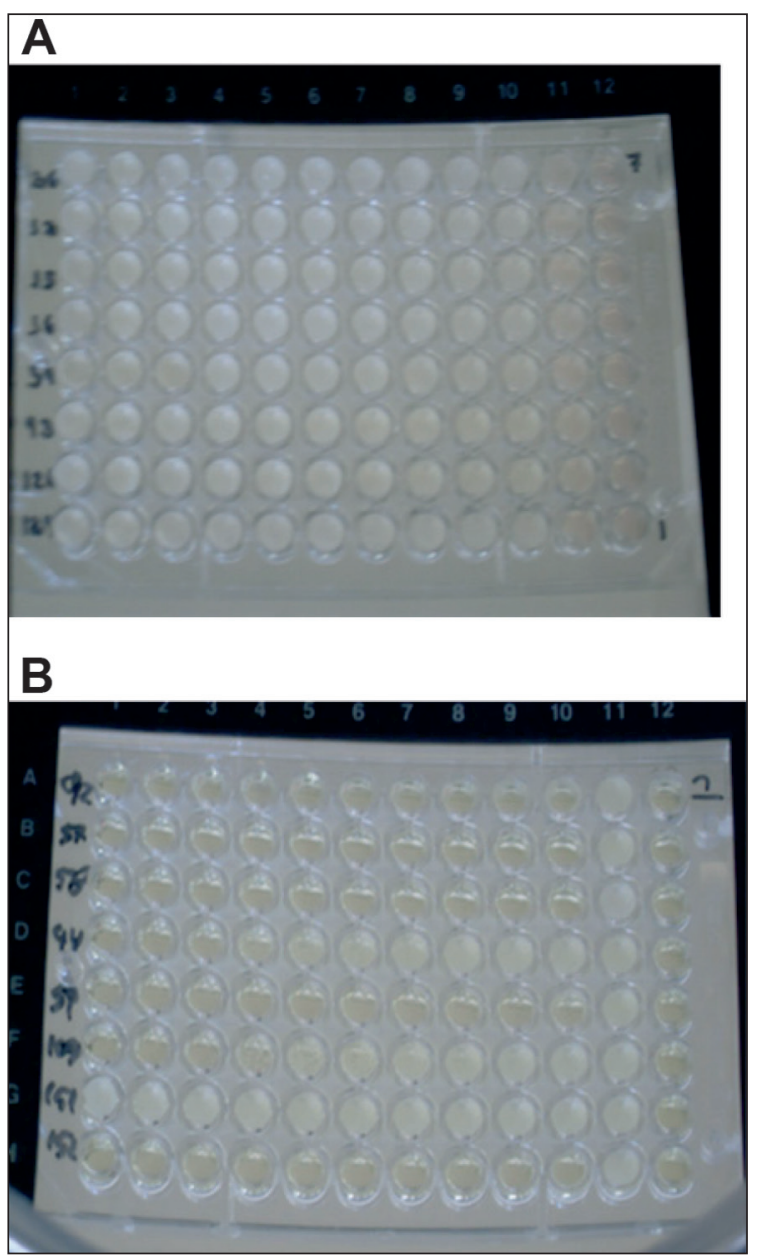

Figura 3. Placa de microdilución. A) a pH 7,0. Se observa turbidez en todos los pocillos, lo cual indica que no hubo inhibición de las cepas a pH 7,0. B) a pH 4,0. La mayoría de las cepas fue inhibida por QAPM a pH ácido.

glabrata). La mayor parte de las cepas de C. glabrata estudiadas fueron inhibidas por QAPM a las concentraciones ensayadas (9/11) (Tabla 3).

\section{Discusión}

En general, con ambos métodos las cepas fueron inhibidas en su mayoría a pH 4,0. Es interesante destacar que todas cepas ensayadas fueron inhibidas a tiempos tan bajos de exposición a QAPM, como un minuto (Tabla 2), indicando que QAPM presenta un efecto anti-fúngico rápido. Además QAPM fue capaz de inhibir a la mayoría de las cepas sensibles dosis dependiente (SDD) y resistentes a fluconazol.

Una posible alternativa de uso terapéutico de QAPM, podría ser en la candidiasis vulvo-vaginal. Esta última, 
es una infección frecuente en la mujer, cuyo agente etiológico más común es $C$. albicans, aunque pueden aislarse otras especies, algunas de ellas, más resistentes a los anti-fúngicos convencionales, como C. glabrata $^{13}$.

Estos resultados demuestran que QAPM tiene un efecto inhibitorio en levaduras del género Candida, incluyendo C. glabrata, y este efecto ocurre a $\mathrm{pH}$ ácido, similar a las condiciones del ambiente vaginal normal o durante una candidiasis vulvo-vaginal, $(\mathrm{pH}=4,0-4,5)^{13}$, podría ser alternativa de tratamiento de uso tópico contra esta patología. Recientes trabajos, han estudiado el uso de quitosán como vehículo de liberación de drogas antifúngicas intravaginales, como econazo ${ }^{14}$. La mayoría de estos estudios se han realizado en tejido vaginal ex vivo.

Este trabajo utilizó 38 cepas clínicas como primera aproximación. Se requiere un estudio que analice un mayor número de cepas y una evaluación del perfil de seguridad de este compuesto al ser aplicado por vía intra-vaginal.

Estos resultados preliminares muestran que QAPM, tendría actividad contra algunas cepas clínicas de Candida sp y que esta actividad se presenta a pH ácido $(4,0)$.

Agradecimientos: Al Curso "Unidades de Investigación" impartido por el Instituto de Ciencias Biomédicas de la Fa- cultad de Medicina de la Universidad de Chile para alumnos de segundo año de Medicina, que permitió la realización de este trabajo.

\section{Resumen}

El quitosán es un polisacárido de D-glucosamina derivado de la quitina que presenta una actividad antimicrobiana frente a bacterias y hongos. Objetivo: Evaluar el efecto antifúngico de quitosán de alto peso molecular (QAPM) en cepas clínicas de Candida sp. Metodología: Se estudiaron 40 cepas (16 Candida albicans, 11 C. glabrata, 5 C. tropicalis, 5 C. krusei, 2 C. parapsilosis y 2 C. famata) mediante un método de microdilución en caldo a pH 7,0 y a ph 4,0. Resultados: De un total de 40 cepas analizadas sólo dos cepas fueron inhibidas por QAPM a $\mathrm{pH} 7,0$ y correspondieron a las cepas control ATCC ( $C$. krusei 6258 y C. parapsilosis 22019). Como contrapatida, $37 / 40$ cepas $(92,5 \%)$ fueron inhibidas a concentraciones inferiores a 1,25 mg/mL de QAPM a pH 4,0. Conclusión: Estos resultados muestran que el QAPM, presenta actividad antifúngica contra cepas clínicas de Candida $s p$., incluyendo $C$. glabrata y que esta actividad ocurre a $\mathrm{pH}$ ácido $(4,0)$. Este compuesto podría utilizase en candidiasis vulvovaginal que cursa a $\mathrm{pH} 4,0-4,5$.

\section{Referencias}

1.- Rabea E I, Badawy M E, Stevens C V, Smagghe G, Steurbaut W.Chitosan as antimicrobial agent: applications and mode of action. Biomacromolecules 2003; 4: 1457-65.

2.- Zakrzewska A, Boorsma A, Brul S, Hellingwerf K J, Klis F M. Transcriptional response of Saccharomyces cerevisiae to the plasma membrane-perturbing compound chitosan. Eukaryot Cell 2005; 4: 703-15.

3.- Reis C P, Ribeiro A J, Veiga F, Neufeld R J, Damgé C. Polyelectrolyte biomaterial interactions provide nanoparticulate carrier for oral insulin delivery. Drug Deliv 2008; 15: 127-39.

4.- Yao H T, Huang S Y, Chiang M T. A comparative study on hypoglycemic and hypocholesterolemic effects of high and low molecular weight chitosan in streptozotocin-induced diabetic rats. Food Chem Toxicol 2008; 46: 1525-34.

5.- Jull A B, Ni Mhurchu C, Bennett D A, Dunshea-Mooij C A, Rodgers A. Chitosan for overweight or obesity. Cochrane Database Syst Rev 2008; 16: CD003892.

6.- Cárdenas G, Anaya P, von Plessing C, Rojas C, Sepúlveda J. Chitosan composite films. Biomedical applications. J Mater Sci Mater Med 2008; 19: 2397-405.

7.- Dhanikula A B, Panchagnula R. Development and characterization of biodegradable chitosan films for local delivery of Paclitaxel. AAPS J 2004; 6: e27.

8.- Baek K S, Won E K, Choung S Y. Effects of chitosan on serum cytokine levels in elderly subjects Arch Pharm Res 2007; 30: 1550-7.

9.- Rhoades J, Roller S. Antimicrobial actions of degradaded and native chitosan against spiolage organisms in laboratory media and foods. Appl Environment Microbiol 2000; 66: 80-6.

10.- Yadav A V, Bhise S B. Chitosan: a potential biomaterial effective against thyphoid. Current Science 2004; 87: 1176-8.
11.- Seyfarth F, Schliemann S, Elsner P, Hipler U C. Antifungal effect of high- and low-molecularweight chitosan hydrochloride, carboxymethyl chitosan, chitosan oligosaccharide and N-acetylD-glucosamine against Candida albicans, Candida krusei and Candida glabrata. Int J Pharm 2008; 353: 139-48.

12.- Subcommittee on antifungal susceptibility testing (ASFT) of the ESCMID European Committee for Antimicrobial susceptibility testing (EUCAST). Method for the derermination of minimum inhibitory concentration (MIC) by broth dilution of fermentative yeasts. Clin Microbiol Infect 2003; 9: 1-8.

13.- Sobel J D. Vulvovaginal candidosis. Lancet 2007; 369 (9577): 1961-71.

14.- Albertini B, Passerini N, Di Sabatino M, Vitali B, Brigidi P, Rodríguez L. Polymer-lipid based mucoadhesive microspheres prepared by spray-congealing for the vaginal delivery of econazole nitrate. Eur J Pharm Sci 2009; 36 (4-5): 591-601. 